\title{
Identification of Differentially Expressed miRNAs between White and Black Hair Follicles by RNA-Sequencing in the Goat (Capra hircus)
}

\section{Zhenyang Wu ${ }^{1}$, Yuhua Fu ${ }^{1}$, Jianhua Cao ${ }^{1}$, Mei Yu ${ }^{1}$, Xiaohui Tang ${ }^{2}$ and Shuhong Zhao ${ }^{1, *}$}

1 Key Laboratory of Agricultural Animal Genetics, Breeding and Reproduction, Ministry of Education, Huazhong Agricultural University, Wuhan 430070, Hubei, China; E-Mails: wuzhenyang0724@gmail.com (Z.W.); yhfu2012@gmail.com (Y.F.); jhcao@mail.hzau.edu.cn (J.C.); yumei@mail.hzau.edu.cn (M.Y.)

2 Agriculture and Animal Husbandry College of Tibet University, Linzhi 860000, Tibet, China; E-Mail: xiaoyuhong515@hotmail.com

* Author to whom correspondence should be addressed; E-Mail: shzhao@mail.hzau.edu.cn; Tel.: +86-27-8738-7480; Fax: +86-27-8728-0408.

Received: 12 April 2014; in revised form: 12 May 2014 / Accepted: 14 May 2014 / Published: 28 May 2014

\begin{abstract}
MicroRNAs (miRNAs) play a key role in many biological processes by regulating gene expression at the post-transcriptional level. A number of miRNAs have been identified from livestock species. However, compared with other animals, such as pigs and cows, the number of miRNAs identified in goats is quite low, particularly in hair follicles. In this study, to investigate the functional roles of miRNAs in goat hair follicles of goats with different coat colors, we sequenced miRNAs from two hair follicles samples (white and black) using Solexa sequencing. A total of 35,604,016 reads were obtained, which included 30,878,637 clean reads (86.73\%). MiRDeep2 software identified 214 miRNAs. Among them, 205 were conserved among species and nine were novel miRNAs. Furthermore, DESeq software identified six differentially expressed miRNAs. Quantitative PCR confirmed differential expression of two miRNAs, miR-10b and miR-211. KEGG pathways were analyzed using the DAVID website for the predicted target genes of the differentially expressed miRNAs. Several signaling pathways including Notch and MAPK pathways may affect the process of coat color formation. Our study showed that the identified miRNAs might play an essential role in black and white follicle formation in goats.
\end{abstract}


Keywords: goat; coat color; RNA-sequencing; hair follicle; miRNA

\section{Introduction}

Coat color has long been a subject of interest to breeders and scientists [1]. Not only is coat color a model phenotype for studying gene action and gene interactions, but also is important for goatskin, which is a valuable animal product. Mammalian coat color is almost totally dependent on either the presence or absence of melanin in skin and follicles [2]. Therefore, it is necessary to understand the process of melanocyte formation. Melanocytes emerge from the neural crest, which is an early embryonic structure [3]. In the late embryonic stage, neural crest cells differentiate into melanoblasts, which migrate to the skin basal layer, where they settle and are involved in the development of the hair follicles [4]. The pigment cells reside in the bulb of the hair follicle and affect the coat color. Various factors affect mammalian coat color, including the composition, numbers, and arrangements of the melanin granules [5]. Moreover, a number of genes such as TYR, MITF, ASIP and MCIR regulate the progress of hair follicle pigmentation. However, few studies have studied the regulatory mechanisms at the post-transcriptional level. MicroRNAs (miRNAs) are small non-protein-coding transcripts that regulate gene expression post-transcriptionally by binding to the 3'-untranslated region (3'-UTR) of the target messenger RNAs (mRNAs) thereby causing suppression of protein synthesis or mRNA cleavage [6]. Increasing evidence shows that miRNAs play an important regulatory role in a variety of biological processes. The development of next-generation massively sequencing (NGMS) technologies, providing high throughput with low cost, have revolutionized genomic research, allowing many animal miRNAs to be identified and deposited in MiRBase (http://www.mirbase.org/). To date, 24,521 entries representing hairpin precursor miRNAs, expressing 30,424 mature miRNAs products in 206 species have been identified and deposited in the public miRNA database miRBase (Release 20.0, June 2013). Among them, 2578 miRNAs were from human, 1908 from mouse, and 153 from sheep. Only a few studies identified miRNAs in goats (Capra hircus) [7-9], indicating that goat miRNAs still need to be sequenced.

In the last two years, significant progress has been made on the goat genome. The $2.66 \mathrm{~Gb}$ genome sequence data were obtained by combing short-read sequencing data and optical mapping data from a female Yunnan black goat. Meanwhile, 51 differentially expressed genes between the two types of hair follicles, the primary and secondary follicle, of a cashmere goat, were identified by comparative transcriptome analysis [10]. Conserved miRNAs (346) were identified between dry period and peak lactation mammary gland tissues in the dairy goat [11]. Five differentially expressed miRNAs were verified by quantitative PCR in the ovaries of pregnant and non-pregnant goats [12]. Hair color is an important trait in the goat. Recently, several studies have tried to identify genes and miRNAs in goatskin and hair follicles. MiRNA data produced by Solexa sequencing among three follicular cycling stages in goatskin and hair follicles were reported [13]. Similar studies focused on the identification of miRNAs in hair follicle and skin development [14-16]. These studies enriched the goat hair follicle and skin miRNA database and enhanced our understanding of the process of miRNA regulation on development of skin and hair follicle. However, very few references are related to the mechanism of 
how miRNAs regulate coat color. Five differentially expressed miRNAs between the white and brown skin of alpaca were identified by quantitative PCR, including miR-211 and miR-202, which were significantly expressed in brown and white skins, respectively [17]. MiR-137, which can downregulate the microphthalmia-associated transcription factor (MITF) was verified to influence the phenotype of coat color in transgenic mice overexpressing miR-137 [18]. No studies have been attempted to identify miRNAs affecting coat color in the goat.

In this study, we sequenced miRNAs from black and white hair follicles collected from 1-year old crossbreed white and black coat color goats. These results provide new information on miRNA expression profiles in the goat and identify possible miRNA regulated pathways related to pigmentation in hair follicles.

\section{Results}

\subsection{Overview of Sequencing Data}

To identify differentially expressed miRNAs in the two types of hair follicles, two small RNA libraries were constructed for Solexa sequencing. A total of 35,604,016 raw reads and 30,878,637 clean reads were obtained after eliminating the low quality reads and adaptor sequences. The size distribution of the reads was similar between the two libraries. Most of the reads were from 21 to $24 \mathrm{nt}$. Reads whose sizes were $22 \mathrm{nt}$ accounted for $20.33 \%$ and $20.16 \%$ of the total sequence reads whereas the reads that were $24 \mathrm{nt}$ accounted for $22.01 \%$ and $19.70 \%$ in white follicle and black follicle libraries, respectively; the size distribution of the small RNAs from white and black hair follicles was similar.

\subsection{Identification of Conserved and Novel MiRNAs}

To identify conserved miRNAs in goat hair follicles, the reads were compared to the precursors and mature miRNAs in miRBase 20.0 (http://www.mirbase.org). In total, 205 and nine conserved and novel miRNAs were identified, respectively. Among these conserved miRNAs, 168 miRNAs were also identified in cattle and only 37 miRNAs in sheep. All the information is shown in Table S1, The 10 most abundant miRNAs are also listed in Table 1. The precursor sequences and secondary structures of the nine novel miRNAs identified from our sequencing data using miRDeep2 software (Table S2) were predicted. The five most abundantly expressed novel miRNAs are shown in Tables 2 and 3, and Figure 1. The expression levels of the novel miRNAs were relatively low in our results. A total of 193 conserved and novel miRNAs were co-expressed, eight miRNAs were white follicle-specific and 13 were black follicle-specific.

Table 1. The most abundantly expressed miRNAs in goat hair follicles.

\begin{tabular}{ccccc}
\hline \multirow{2}{*}{ MiRNAs name } & \multicolumn{2}{c}{ Normalized expression level } & \multirow{2}{*}{ Mature sequences } \\
\cline { 2 - 3 } & WF & BF & \\
\hline Goat-miR-146b-5p & $186,997.77$ & $158,761.10$ & ugagaacugaauuccauaggcugu \\
Goat-miR-27b-3p & $79,872.78$ & $72,800.46$ & uucacaguggcuaaguucugc \\
Goat-miR-205-5p & $20,575.80$ & $19,911.95$ & & uccuucauuccaccggagucug \\
\hline
\end{tabular}


Table 1. Cont.

\begin{tabular}{|c|c|c|c|}
\hline \multirow{2}{*}{ MiRNAs name } & \multicolumn{2}{|c|}{ Normalized expression level } & \multirow{2}{*}{ Mature sequences } \\
\hline & WF & BF & \\
\hline Goat-miR-181a-2-5p & $21,177.16$ & $16,613.29$ & aacauucaacgcugucggugagu \\
\hline Goat-miR-181a-1-5p & $21,176.79$ & $16,613.08$ & aacauucaacgcugucggugagu \\
\hline Goat-miR-92a-3p & $19,003.38$ & $17,003.44$ & uauugcacuugucccggccugu \\
\hline Goat-miR-182-5p & $14,218.79$ & $13,630.30$ & uuuggcaaugguagaacucacacu \\
\hline Goat-miR-26a-1-5p & $14,855.58$ & $12,171.42$ & uucaaguaauccaggauaggcu \\
\hline Goat-miR-26a-2-5p & $14,837.64$ & $12,152.12$ & uucaaguaauccaggauaggcu \\
\hline Goat-let-7f-5p & $10,685.28$ & 8870.12 & ugagguaguagauuguauaguu \\
\hline
\end{tabular}

Table 2. The five most abundantly expressed novel miRNAs in goat hair follicels.

\begin{tabular}{|c|c|c|c|}
\hline \multirow{2}{*}{ MiRNAs name } & \multicolumn{2}{|c|}{ Normalized expression level } & \multirow{2}{*}{ Mature sequences } \\
\hline & WF & BF & \\
\hline Novel-84-3p & 195.35 & 203.44 & uccccugcaucuccacca \\
\hline Novel-9-3p & 94.46 & 84.20 & aaaaccugaaugaacuuuugag \\
\hline Novel-21-3p & 58.89 & 53.72 & aaagccugaaugaacuuuuugg \\
\hline Novel-29-5p & 105.15 & 0 & aagguagauagaacaggucuug \\
\hline Novel-76-5p & 42.67 & 40.61 & uauugcacauuacuaaguugc \\
\hline
\end{tabular}

Table 3. Predicted precursor sequences and genome locations of five novel miRNAs.

\begin{tabular}{|c|c|c|c|}
\hline MiRNAs name & MiRNA precursor sequence & Chromosome & Strand \\
\hline Novel-84-3p & $\begin{array}{l}\text { ggcuacagucugugggguugcagaguuggaca } \\
\text { caacugagcacaucccugcaucuccacca }\end{array}$ & chr8 & - \\
\hline Novel-9-3p & $\begin{array}{l}\text { aaaauguucaugcagguuuuuccguaagauguu } \\
\text { acaggaaaaccugaaugaacuuuugag }\end{array}$ & $\operatorname{chrX}$ & + \\
\hline Novel-21-3p & $\begin{array}{l}\text { caauaaguucguuuggguuuuuggcuguuacag } \\
\text { aaagccugaaugaacuuuuugg }\end{array}$ & $\operatorname{chrX}$ & + \\
\hline Novel-29-5p & $\begin{array}{l}\text { aagguagauagaacaggucuugugugcaaaaugaa } \\
\text { uucaagaccuacuuaucuaccaacagc }\end{array}$ & $\operatorname{chr} 21$ & + \\
\hline Novel-76-5p & $\begin{array}{l}\text { uauugcacauuacuaaguugcauguugucacggcc } \\
\text { ucagugcaauuuagugugugugauauu }\end{array}$ & chr8 & - \\
\hline
\end{tabular}

WF: white follicles and BF: black follicle.

\subsection{Identification of Differentially Expressed MiRNAs and Prediction of MiRNA Target Genes,} Pathways, and GO Analysis

The DESeq package analyzed the differentially expressed miRNAs and identified six miRNAs. Five miRNAs in the white hair follicle were significantly upregulated, while one miRNA significantly downregulated (Figure 2). Normalized expression levels in the white and black hair follicles of the differentially expressed miRNAs are listed in Table 4. 
Figure 1. Predicted secondary structures of novel miRNAs. The red color indicates the mature sequence, the yellow color indicates the loop sequence, the blue color indicates the predicted star sequence, and the purple indicates the miRNA star sequences.

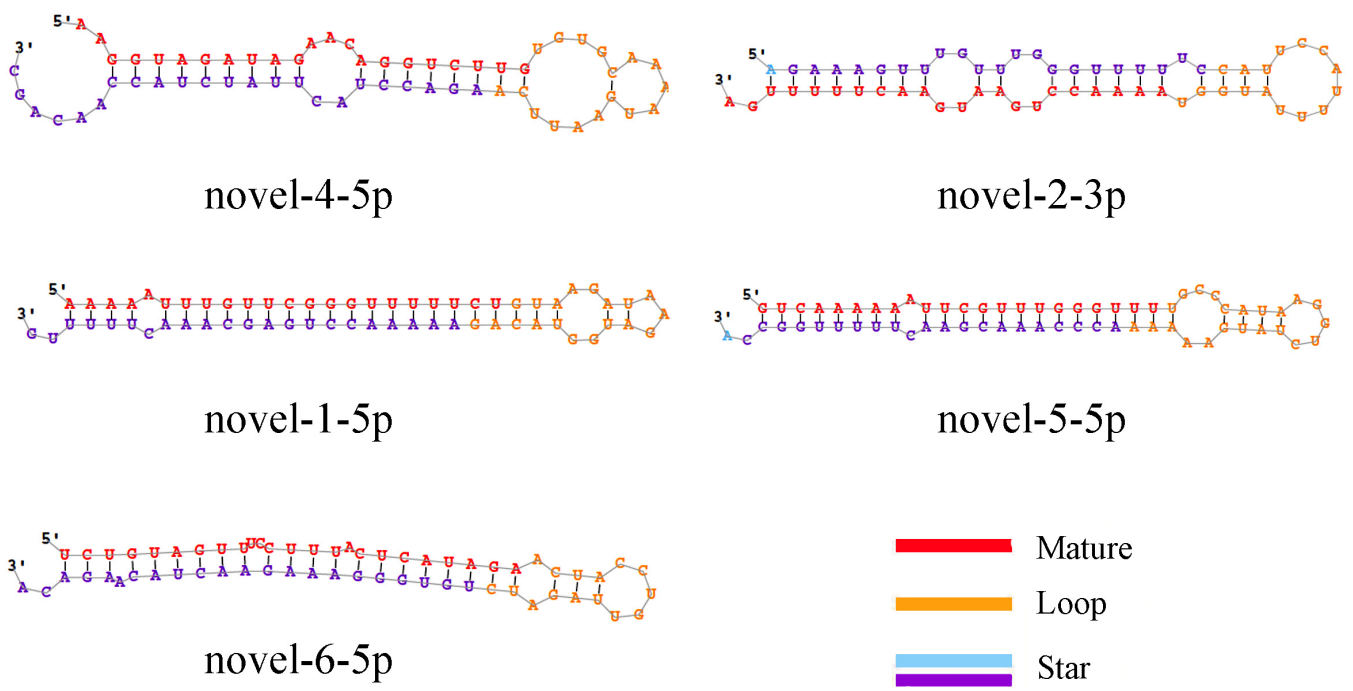

Figure 2. Plot of normalized mean versus log2-fold change for the miRNAs in black and white hair follicles. The red colour marks genes detected as differentially expressed. The red points and triangle below and above the red line indicate downregulation and upregulation, respectively in black hair follicles, and the reverse in white hair follicles. The symbols at the upper and lower plot border (the red and dark triangle) indicate genes with very large or infinite log fold change.

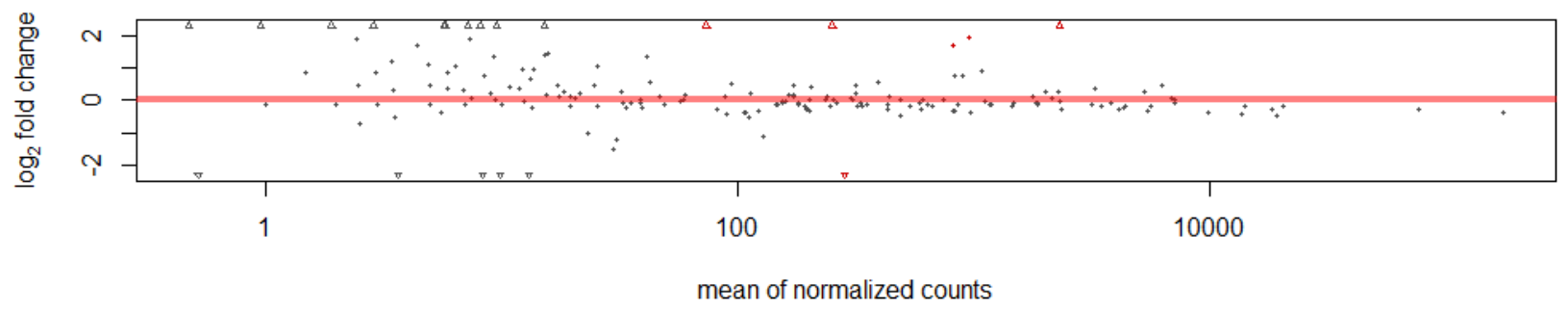

Table 4. Differentially expressed miRNAs among different goat follicle tissues.

\begin{tabular}{cccc}
\hline \multirow{2}{*}{ MiRNAs } & \multicolumn{2}{c}{ Normalized expression level } & \multirow{2}{*}{$\boldsymbol{p}$ value } \\
\cline { 2 - 3 } & WF & BF & \\
\hline Goat-miR-10b-5p & 0 & $64,534.56$ & $2.36 \times 10^{-121}$ \\
Goat-miR-1307-5p & 7796.83 & 0 & $6.98 \times 10^{-25}$ \\
Goat-miR-146a-5p & 0 & 2054.46 & $2.77 \times 10^{-7}$ \\
Goat-miR-143-3p & 5469.10 & $20,954.76$ & $1.14 \times 10^{-5}$ \\
Goat-miR-30a-5p & 5363.49 & $17,292.17$ & $2.69643 \times 10^{-4}$ \\
Goat-miR-211-5p & 0 & 7016.16 & $3.84 \times 10^{-22}$ \\
\hline
\end{tabular}

To understand the function of the differentially expressed miRNAs in goat hair follicles, we predicted the target genes of the miRNAs using The Target Scan website, PicTar and DIANA-microT v3.0, and counted miRNAs that were predicted by two of these software tools. As a result, 981 genes were found to be targeted by five differentially expressed miRNAs, except miR-1307 (Table S3). These genes 
were analyzed by the DAVID website, identifying 26 pathways that could be involved in regulation of coat color (Table S4). We listed the 20 pathways for which the gene count was more than $1 \%$ in Figure 3. Among these pathways, the mitogen-activated protein kinase (MAPK) signaling pathway regulates cell proliferation and differentiation, and can induce MITF, which increases the production of tyrosinase [19]. The MAPK signaling pathway is involved in 27 of the genes, which are targeted by the five miRNAs (Table 5).

Figure 3. Top 20 pathways predicted to be targeted by differentially expressed miRNAs.

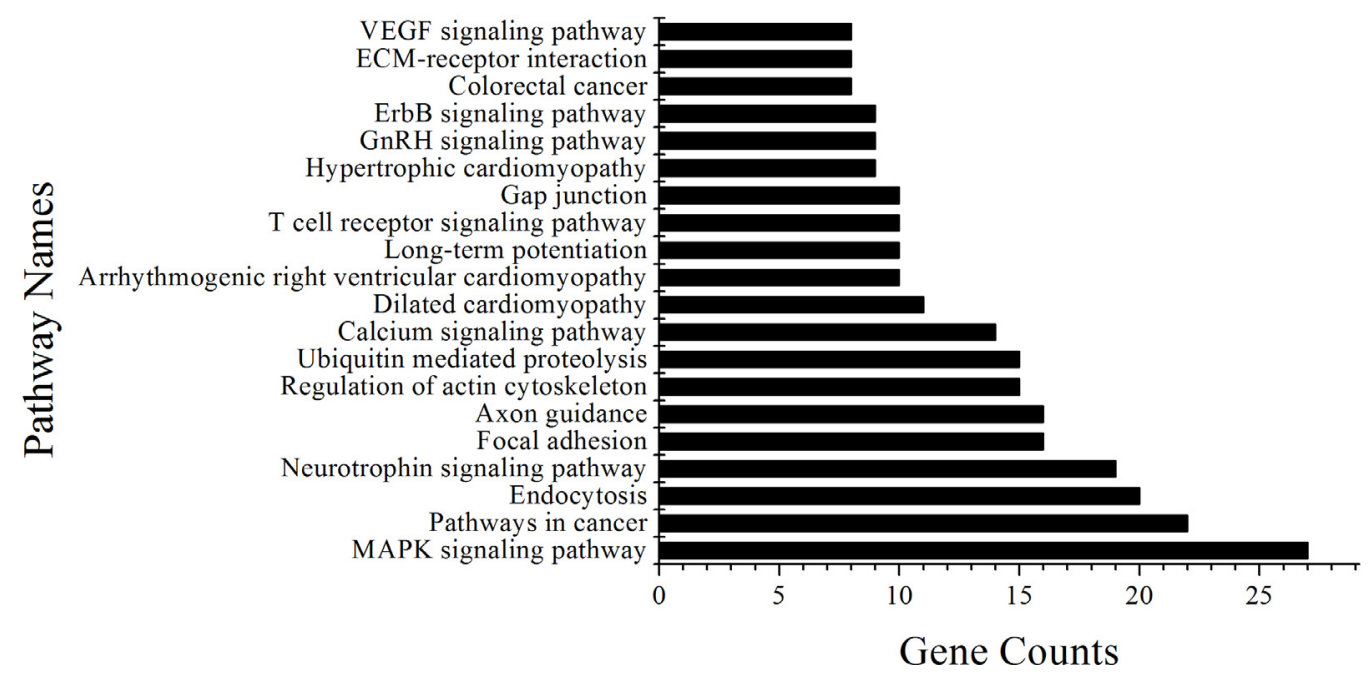

Table 5. Target gene of differentially expressed miRNAs in the MAPK signaling pathway.

\begin{tabular}{cc}
\hline MiRNA name & Target gene in MAPK signaling pathway \\
\hline MiR-10b & $B D N F$ \\
MiR-211 & $S O S 1$ \\
MiR-143 & $C A C N A 1 E, F G F 1, M A P K 7, M A P 3 K 7, P D G F R A, K R A S$ \\
& $R A P 1 B, R A S A 1, R A P G E F 2, T A O K 1, C A C N B 2, C A S P 3$, \\
MiR-30a & $C A C N A 1 C, I L 1 A, M A P 2 K 4, M A P 3 K 1, M A P 3 K 12, M A P 3 K 2$, \\
& $M A P 3 K 5, N F 1, P P P 3 C A, P P P 3 C B, R P S 6 K A 2, C R K L$ \\
MiR-146a & $T R A F 6$ \\
\hline
\end{tabular}

To gain an insight into the molecular functions of genes in biological processes, we annotated the genes targeted by differentially expressed miRNAs using GO categories (Table S5). The five most enriched GO categories contained at least $10 \%$ of the total predicted target genes in the biological processes analysis. Most genes were enriched in two types of functions for molecular function: binding activity and transcription activity. The 10 most enriched GO categories are listed in Tables 6-8.

Expression of tyrosinase gene (TYR) and related genes, such as tyrosinase-related proteins-1 (TYRP1), is the hallmark of mammalian melanocytes [20]. The biosynthesis of the pigment itself is a process orchestrated by the concerted action of tyrosinase, tyrosinase-related protein-1 and dopachrome tautomerase. They are melanocyte-specific enzymes that act to synthesize two types of pigment, eumelanin (black/brown) and/or pheomelanin (yellow/red), which are then deposited in discrete membrane-bound organelles, known as melanosomes [21,22]. The proportion of the two types 
of pigment result in different coat colors, such as red, yellow, brown or white. If the process of pigment synthesize are blocked directly or indirectly, the coat color will change.

The possible regulatory pathways of the differentially expressed miRNAs were built according to the results from our study and a literature search (Figure 4).

Table 6. The 10 most enriched GO categories in biological process.

\begin{tabular}{lllll}
\hline GO accession & GO terms & Gene count & Percentage $(\%)$ & $\boldsymbol{p}$ value \\
\hline GO:0006357 & regulation of transcription & 188 & 23.76738306 & $1.04 \times 10^{-10}$ \\
GO:0045449 & transcription & 151 & 19.0897598 & $2.51 \times 10^{-8}$ \\
GO:0010557 & regulation of RNA metabolic process & 128 & 16.18204804 & $1.18 \times 10^{-6}$ \\
GO:0045893 & regulation of transcription, DNA-dependent & 125 & 15.80278129 & $1.77 \times 10^{-6}$ \\
GO:0051254 & intracellular signaling cascade & 86 & 10.87231353 & $2.94 \times 10^{-4}$ \\
& regulation of transcription from RNA & 79 & 9.987357775 & $3.36 \times 10^{-12}$ \\
GO:0031328 & polymerase II promoter & & & \\
& positive regulation of macromolecule & 78 & 9.860935525 & $1.89 \times 10^{-8}$ \\
GO:0010629 & metabolic process & 69 & 8.723135272 & $1.28 \times 10^{-6}$ \\
GO:0009891 & regulation of apoptosis & 69 & 8.723135272 & $1.81 \times 10^{-6}$ \\
GO:0045941 & regulation of programmed cell death & 69 & 8.723135272 & $2.06 \times 10^{-6}$ \\
GO:0010628 & regulation of cell death & & & \\
\hline & & & & \\
\end{tabular}

Table 7. The 10 most enriched GO categories in cellular component.

\begin{tabular}{lllll}
\hline GO accession & GO terms & Gene count & Percentage $(\%)$ & $\boldsymbol{p}$ value \\
\hline GO:0043232 & intracellular non-membrane-bounded & 132 & 16.687737 & 0.00202852 \\
& organelle & 132 & 16.687737 & 0.00202852 \\
GO:0043228 & non-membrane-bounded organelle & 110 & 13.9064475 & 0.009611689 \\
GO:0044459 & plasma membrane part & 99 & 12.5158028 & 0.002133467 \\
GO:0031974 & membrane-enclosed lumen & 97 & 12.2629583 & 0.00251202 \\
GO:0043233 & organelle lumen & 96 & 12.136536 & 0.001867367 \\
GO:0070013 & intracellular organelle lumen & 90 & 11.3780025 & $2.40 \times 10^{-5}$ \\
GO:0031981 & nuclear lumen & 78 & 9.86093552 & $4.47 \times 10^{-7}$ \\
GO:0000267 & cell fraction & 70 & 8.84955752 & $7.38 \times 10^{-9}$ \\
GO:0005626 & insoluble fraction & 69 & 8.72313527 & $3.94 \times 10^{-9}$ \\
GO:0005624 & membrane fraction & & & \\
\hline
\end{tabular}

Table 8. The 10 most enriched GO categories in molecular function.

\begin{tabular}{lllll}
\hline GO accession & GO terms & Gene count & Percentage (\%) & $\boldsymbol{p}$ value \\
\hline GO:0043167 & ion binding & 242 & 31 & $8.45 \times 10^{-4}$ \\
GO:0046872 & metal ion binding & 240 & 30 & $3.30 \times 10^{-4}$ \\
GO:0043169 & cation binding & 240 & 30 & $6.09 \times 10^{-4}$ \\
GO:0046914 & transition metal ion binding & 167 & 21 & 0.001082879 \\
GO:0003677 & DNA binding & 156 & 20 & $7.00 \times 10^{-6}$ \\
GO:0008270 & zinc ion binding & 146 & 18 & $2.68 \times 10^{-4}$ \\
GO:0000166 & nucleotide binding & 132 & 17 & 0.008790377 \\
GO:0030528 & transcription regulator activity & 126 & 16 & $4.59 \times 10^{-10}$ \\
GO:0003700 & transcription factor activity & 85 & 11 & $9.09 \times 10^{-8}$ \\
GO:0043565 & sequence-specific DNA binding & 50 & 6.3 & $2.57 \times 10^{-4}$ \\
\hline
\end{tabular}


Figure 4. The possible regulatory pathway of melanogesis activation.

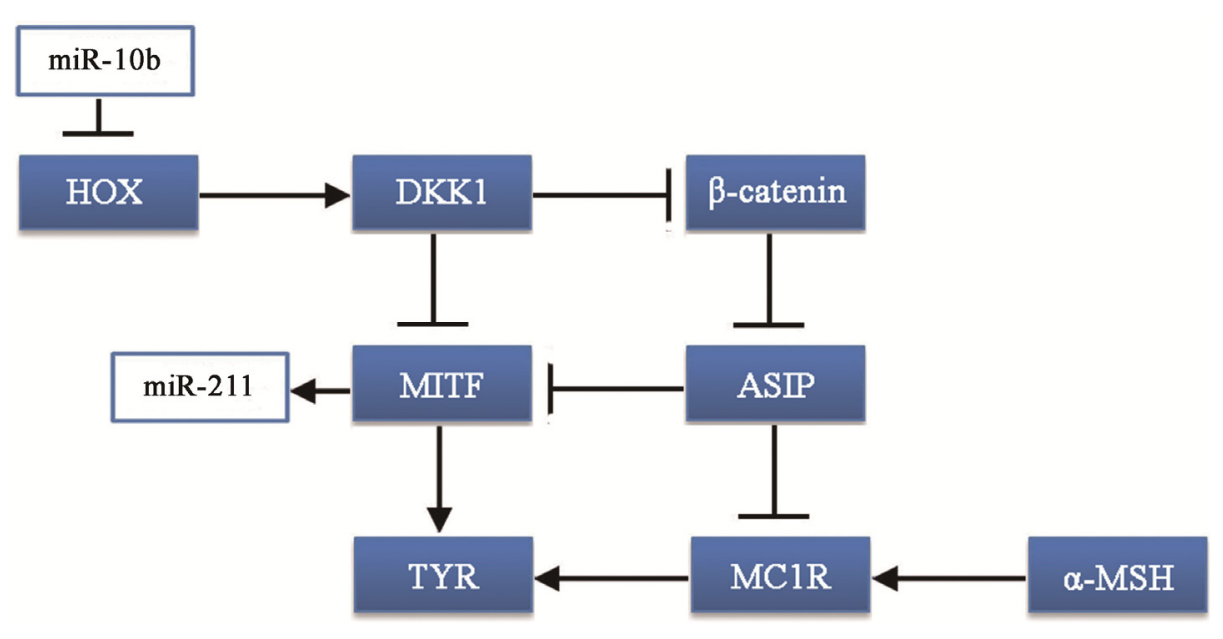

\subsection{Validation of the Sequencing Data by Quantitative PCR ( $P C C R)$}

To verify the sequencing results, the differentially expressed miRNAs were detected by qPCR analyses. The expressions of two miRNAs, miR-10b and miR-211, were confirmed (Figure 5). The result is consistent with the sequencing data. The expression levels of miR-10b and miR-211 were significantly higher in black follicles than in white follicles.

Figure 5. The expression of miR-211 and miR-10b in two tissues. MiR-211 is highly expressed in black hair follicle (BF) compared with the white hair follicle (WF). Results are the mean $\pm \mathrm{SD}$ from triplicate determinations. $p<0.01(* *)$. MiR-10b is highly expressed in black hair follicles (BF) in compared with the white hair follicles (WF). Results are the mean \pm SD from triplicate determinations. $p<0.05(*)$.

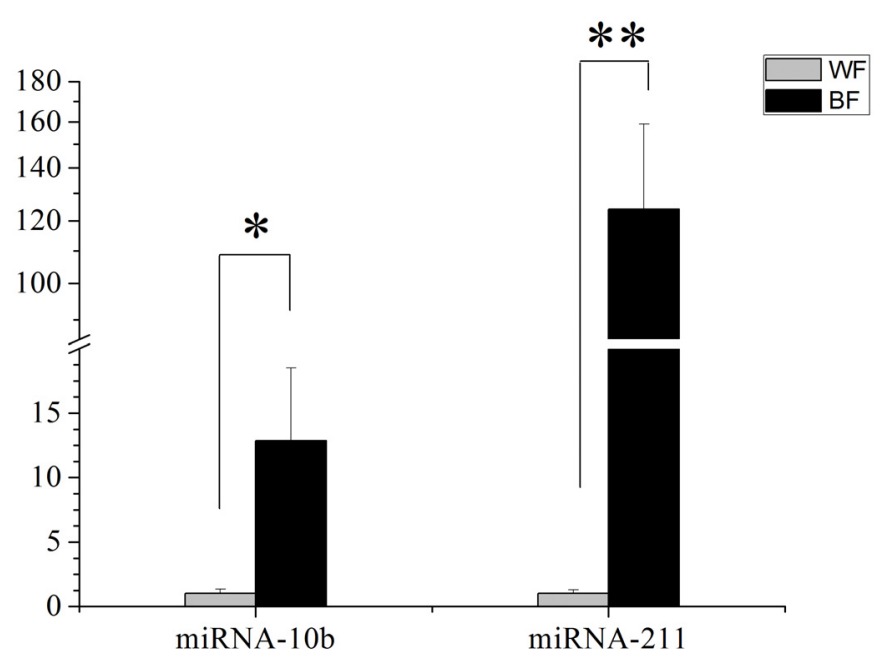

\section{Discussion}

This study identified 205 conserved miRNAs and nine novel miRNAs by RNA-Seq in goat hair follicles. Six differentially expressed miRNAs were predicted in two types of hair follicles tissues. Most miRNAs were upregulated in black follicles, only miR-1307 was downregulated in white hair follicle. However, the expression of the novel miRNA was too low to perform further detection. Two 
differentially expressed miRNAs, miR-10b and miR-211, were verified by qPCR. Our results thus offer new information on goat hair follicle expressed miRNAs.

We obtained 214 miRNAs in this study. However, only a few miRNAs were differentially expressed between white and black hair follicles. One reason may be that the samples we used were from adult goats. Most of the relevant biological processes such as melanoblast differentiation, migration and maturation are completed during embryonic development. Thus, more differentially expressed miRNAs might be identified during early development than in the adult hair follicles where miRNAs may be involved in fewer biological events. In adult hair follicles, genes and miRNAs are probably involved in more functions related to melanocyte stem cell differentiation into melanocytes or the proliferation of melanocytes. Nevertheless, the identification of miRNAs in skin add a new dimension in the regulatory networks and identified novel players in hair follicle color formation [23]. Our results may prompt further studies on how miRNAs affect hair follicle development, differentiation and pigmentation.

In our study, the MAPK signaling pathway was the major pathway involving 27 genes, and targeted by five differentially expressed miRNAs including miR10b. The MAPK family proteins, such as p38, ERK and JNK, play critical roles in melanogenesis [24]. Most studies reported that the p38 MAPK signaling pathway activates MITF, which can up-regulate the expression of melanogenic enzymes [25]. However, the ERK and/or JNK/SAPK pathways cause down-regulation of melanin synthesis by downregulating MITF [26]. The detailed mechanism involving p38 MAPK in melanin synthesis is not completely understood.

MiR-10b was one of the most abundant and differentially expressed miRNA in black hair follicles, with approximately 64,534.56 reads. MiR-10b takes part in carcinogenesis: miR-10b can suppress the translation of the HOXD10 gene leading to increased RHOC expression and AKT phosphorylation [27,28]. Although currently there are no studies on the impact of the HOXD10 gene on melanocytes or the production of melanin, many studies show that genes in the HOX gene family are related to the development of hair follicles, especially HOXC13 [29,30]. Thus, the HOX gene family may be associated with coat color formation. Moreover, the HOXA10 gene can upregulate the Dickkopf 1 (DKK1) gene [31], which regulates skin pigmentation. DKK1 can inhibit the function and proliferation of melanocytes by suppressing $\beta$-catenin and microphthalmia-associated transcription factor (MITF) [32,33], which can promote the synthesis of melanin.

Our results showed that miR-10b regulates the DVL3 gene in the Notch pathway. Notch is an evolutionarily conserved local cell-signaling pathway that participates in a variety of cellular processes such as cell fate specification, differentiation, proliferation, apoptosis, adhesion, epithelial-mesenchymal transition, migration and angiogenesis [34], and the development of hair follicles [35]. Melanocytes produce melanin and are tightly linked with hair regeneration cycles [36]. In the hair follicle, melanocyte and melanocytes stem cells numbers are maintained in a dynamic balance. In the cell cycle of the hair follicle, melanocytes proliferate during the hair growth phase and are depleted during the regression phase; the new melanocyte is produced by the differentiation and proliferation of melanocyte stem cells [37-39]. The Notch signaling pathway plays a key role in melanoblasts, melanocyte stem cells, keratinocytes and melanocytes [40]. Many studies reported that lack of Notch signaling can lead to the reduction of the number of melanocytes which can cause the coat color [41-43]. Interestingly, HOX, 
the Notch signaling pathway and the Wnt/ $\beta$-catenin signaling pathway interact via cross-talk $[44,45]$. Taken together, miR-10b could be an important regulator in goat coat color formation.

MiR-211 had a similar expression pattern to miR-10b. Most studies of this miRNA are on cancer, with few reports on hair follicle development or coat color. One study reported that miR-211 is highly expressed in brown alpaca skin via white alpaca skin expression [17]. That result is consistent with our study, however, the mechanism has not been investigated. MITF promotes the expression of many genes in pigment cell and regulates melanocyte development by increasing the expression of enzymes, involved in melanin synthesis and melanosome biogenesis [46]. Studies have shown that miR-211 is induced by the expression of MITF [47,48]. This could explain why the expression of miR-211 is higher in black follicles than in white follicles. However, how miR-211 regulates melanocytes or melanin synthesis is not clear.

\section{Experimental Section}

\subsection{Sample Collection, RNA Extraction and Library Construction}

To eliminate the genetic background, samples were collected from three 1-year old crossbreed white and black coat colored goats (Figure 6). Black hair and white hair were pulled out with their follicles, the hair shaft was removed with scissors and transferred to $1.5 \mathrm{~mL}$ RNase-free tube with $1 \mathrm{~mL}$ Trizol reagent (Life Technologies Corporation, Carlsbad, CA, USA). The samples were homogenized and then stored at $-80^{\circ} \mathrm{C}$.

Total RNA was isolated from hair follicles of goats according to the manufacturer's protocol. Quality and quantity of RNA was examined using a NanoDrop 2000/2000C (Thermo Fisher Scientific Inc., Waltham, MA, USA) and integrity was detected using agarose gel electrophoresis. Two samples (one from white hair follicle tissue and one from black hair follicle tissue) from one goat each were sent to Genergy Biotechnology Co., Ltd. (Shanghai, China) for small RNA library construction and sequencing. Sequencing was performed using an Illumina HiSeq 2000 Genome Analyzer (Illumina Inc., Santiago, CA, USA). Libraries were constructed using a TruSeq Small RNA Sample Preparation kit (Illumina Inc., Santiago, CA, USA).

Figure 6. The white and black hair follicles were collected from three 1-year old crossbred black and white goats.
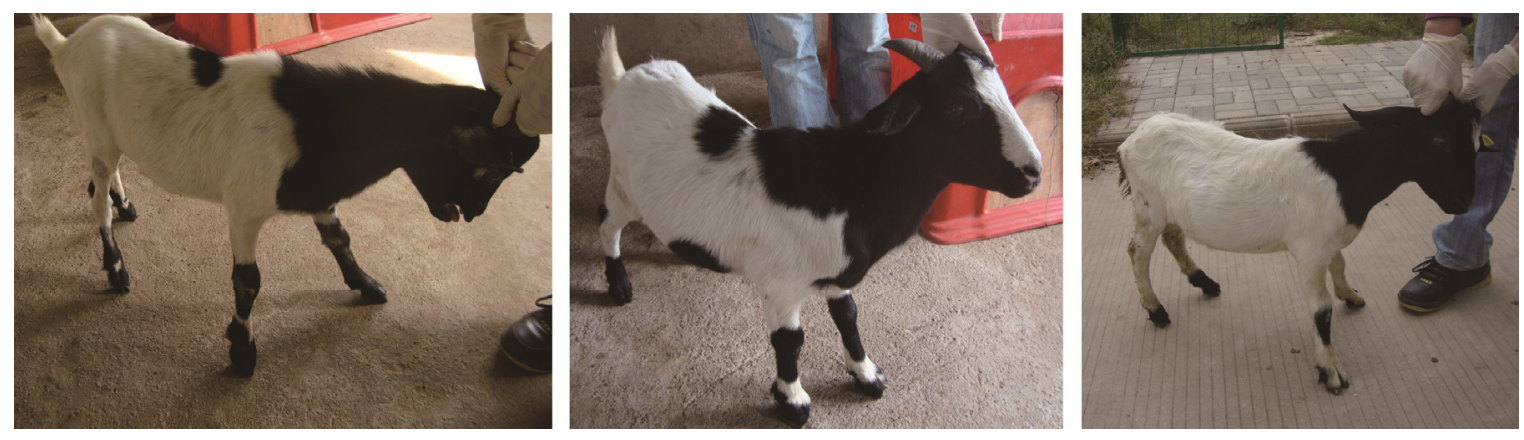


\subsection{Identification of Conserved and Novel MiRNAs}

The quality of the original raw sequencing data obtained by Solexa deep sequencing was assessed using FastQC software (http://www.bioinformatics.babraha m.ac.uk/projects/fastqc/). The clean reads were obtained by trimming the low-quality reads and eliminating reads with contaminants, e.g., reads without 3 ' primer and reads shorter than $18 \mathrm{nt}$. MiRDeep2 software [49,50] was used to predict the known and novel miRNAs. The parameter used to screen for "novel" miRNAs predicted using miRDeep2 were as follows: (a) Delete miRDeep2 score: <100; (b) The ratio of mature miRNA vs. miRNA*; and (c) we screened the predicted miRNAs strictly according to the hairpin structure, with only a 2-nt overhang, which is the hallmark of a bona fide miRNA.

The expression level of each miRNA was normalized by the following formula: Normalized expression $(\mathrm{NE})=$ Actual miRNA count/Total count of clean reads $\times 1,000,000$. We removed the miRNAs with a normalized expression level lower than 1 and an estimated probability value lower than 0.95 .

\subsection{Identification and Validation of Differentially Expressed MiRNAs}

The package DESeq [51] was used to identify differentially expressed miRNAs. This software provides methods to detect miRNA differential expression using the negative binomial distribution and a shrinkage estimator for the distribution's variance.

Differentially expressed miRNAs were confirmed with qPCR [52]. Six samples (three white follicle samples and three black follicle samples) from three goats were used in qPCR analysis. The miRNA specific primers are shown in Table 9. One microgram of total RNA from each sample was reverse-transcribed into cDNA using the Thermo Scientific Revert Aid First Strand cDNA Synthesis Kit (Thermo Fisher Scientific Inc., Waltham, MA, USA). U6 snRNA was used as the internal control. QPCR was performed using standard protocols on the Roche LightCycler 480 Real-Time PCR Detection System (Hoffmann-La Roche Ltd, Basel, Switzerland). The $2^{-\Delta \Delta C t}$ method was used to analyze the expression levels [53].

Table 9. MiRNA and gene primers.

\begin{tabular}{cl}
\hline \multicolumn{1}{c}{ MiRNA } & \multicolumn{1}{c}{ Primer sequences } \\
\hline \multirow{3}{*}{ Goat-miR-211 } & $\begin{array}{l}\text { Forward: TCGGCAGGTCCCTTTGTCATCC } \\
\text { Reverse: TGCAGGTCAACTGGTGTCGT } \\
\text { Loop prime: CTCAACTGGTGTCGTGGAGTCGGCAATTCAGTTGAGTGGGCAAA }\end{array}$ \\
\hline \multirow{2}{*}{ Goat-miR-10b } & $\begin{array}{l}\text { Forward: TCGGCAGGACCCTGTAGAACCG } \\
\text { Reverse: TGCAGGTCAACTGGTGTCGT } \\
\end{array}$ \\
\hline \multirow{2}{*}{ Loop prime: CTCAACTGGTGTCGTGGAGTCGGCAATTCAGTTGAGCACAAATT } \\
\hline
\end{tabular}

\subsection{Prediction of MiRNA Target Genes, Pathways and GO Analysis}

The TargetScan website (http://www.targetscan.org/), PicTar (http://pictar.mdc-berlin.de/) and DIANA-microT v3.0 (http://diana.cslab.ece.ntua.gr/microT/) were used to predict the targets of 
differentially expressed miRNAs The DAVID website [54,55] was used to analyze the KEGG pathways, with the following parameters: Count $=2$ and EASE $=0.1$. GO categories were also analyzed using the DAVID website with the following parameters: Count $=10$ and $\mathrm{EASE}=0.01$.

\section{Conclusions}

In conclusion, the development of hair follicles and melanocytes is a complex event involving numerous genes and pathways that interact and show cross-talk. We showed evidence that miRNAs could also be involved in these processes. Understanding the regulatory mechanism at the post-transcription level will provide new insights into the regulation of coat color formation.

\section{Acknowledgments}

This study is supported by the National Nature Scientific Foundation of China (NO. 31360534), the International collaborating grants from National ministry of science and technology projects of China (NO. 1111), National High Technology Research and Development Program ("863"Program) of China (2013AA102506).

\section{Author Contributions}

Zhenyang Wu: RNA extraction, data analysis and prepared the manuscript. Yuhua Fu and Jianhua Cao: Helped with data analysis and produce some figures. Xiaohui Tang: Sample collection. Shuhong Zhao and Mei Yu: Conceived and designed the experimentand provided funding.

\section{Conflicts of Interest}

The authors declare no conflict of interest.

\section{References}

1. Thiruvenkadan, A.K.; Kandasamy, N.; Panneerselvam, S. Coat colour inheritance in horses. Livest. Sci. 2008, 117, 109-129.

2. Klungland, H.; Våge, D. Pigmentary switches in domestic animal species. Ann. N. Y. Acad. Sci. 2003, 994, 331-338.

3. Paus, R.; Müller-Röver, S.; van der Veen, C.; Maurer, M.; Eichmüller, S.; Ling, G.; Hofmann, U.; Foitzik, K.; Mecklenburg, L.; Handjiski, B. A comprehensive guide for the recognition and classification of distinct stages of hair follicle morphogenesis. J. Investig. Dermatol. 1999, 113, 523-532.

4. Stenn, K.; Paus, R. Controls of hair follicle cycling. Physiol. Rev. 2001, 81, 449-494.

5. Kim, S.C.; Lee, J.H.; Kim, M.H.; Lee, J.A.; Kim, Y.B.; Jung, E.; Kim, Y.S.; Lee, J.; Park, D. Hordenine, a single compound produced during barley germination, inhibits melanogenesis in human melanocytes. Food Chem. 2013, 141, 174-181.

6. Bartel, D.P. MicroRNAs: Target recognition and regulatory functions. Cell 2009, 136, 215-233.

7. Chen, H.-X. Computational and experimental identification of novel microRNAs in goat. Hereditas (Beijing) 2008, 30, 1326-1332. 
8. Wenguang, Z.; Jianghong, W.; Jinquan, L.; Yashizawa, M. A subset of skin-expressed microRNAs with possible roles in goat and sheep hair growth based on expression profiling of mammalian microRNAs. J. Integr. Biol. 2007, 11, 385-396.

9. Lin, X.Z.; Luo, J.; Zhang, L.P.; Zhao, W.S.; Wang, W.; Gou, D.M. Screening miRNAs regulating fatty acid metabolism in goat (Capra hirus) mammary gland and cloning determination of related pri-miRNAs. J. Agric. Biotechnol. 2012, 20, 589-598.

10. Dong, Y.; Xie, M.; Jiang, Y.; Xiao, N.; Du, X.; Zhang, W.; Tosser-Klopp, G.; Wang, J.; Yang, S.; Liang, J.; et al. Sequencing and automated whole-genome optical mapping of the genome of a domestic goat (Capra hircus). Nat. Biotechnol. 2013, 31, 135-141.

11. Li, Z.; Lan, X.; Guo, W.; Sun, J.; Huang, Y.; Wang, J.; Huang, T.; Lei, C.; Fang, X.; Chen, H. Comparative transcriptome profiling of dairy goat microRNAs from dry period and peak lactation mammary gland tissues. PLoS One 2012, 7, e52388.

12. Zhang, X.-D.; Zhang, Y.-H.; Ling, Y.-H.; Liu, Y.; Cao, H.-G.; Yin, Z.-J.; Ding, J.-P.; Zhang, X.-R. Characterization and differential expression of microRNAs in the ovaries of pregnant and non-pregnant goats (Capra hircus). BMC Genomics 2013, 14, 157.

13. Yuan, C.; Wang, X.; Geng, R.; He, X.; Qu, L.; Chen, Y. Discovery of cashmere goat (Capra hircus) microRNAs in skin and hair follicles by Solexa sequencing. BMC Genomics 2013, 14, 511.

14. Geng, R.; Yuan, C.; Chen, Y. Exploring differentially expressed genes by RNA-Seq in cashmere goat (Capra hircus) skin during hair follicle development and cycling. PLoS One 2013, 8, e62704.

15. Liu, Y.; Li, M.; Zhang, G.; Pang, Z. MicroRNA-10b overexpression promotes non-small cell lung cancer cell proliferation and invasion. Eur. J. Med. Res. 2013, 18, 41-49.

16. Liu, Z.; Xiao, H.; Li, H.; Zhao, Y.; Lai, S.; Yu, X.; Cai, T.; Du, C.; Zhang, W.; Li, J. Identification of conserved and novel microRNAs in cashmere goat skin by deep sequencing. PLoS One 2012, 7, e50001.

17. Tian, X.; Jiang, J.; Fan, R.; Wang, H.; Meng, X.; He, X.; He, J.; Li, H.; Geng, J.; Yu, X.; et al. Identification and characterization of microRNAs in white and brown alpaca skin. BMC Genomics 2012, 13, 555.

18. Dong, C.; Wang, H.; Xue, L.; Dong, Y.; Yang, L.; Fan, R.; Yu, X.; Tian, X.; Ma, S.; Smith, G.W. Coat color determination by miR-137 mediated down-regulation of microphthalmia-associated transcription factor in a mouse model. RNA 2012, 18, 1679-1686.

19. Ahn, J.H.; Jin, S.H.; Kang, H.Y. LPS induces melanogenesis through p38 MAPK activation in human melanocytes. Arch. Dermatol. Res. 2008, 300, 325-329.

20. Wang, R.; Tang, P.; Wang, P.; Boissy, R.E.; Zheng, H. Regulation of tyrosinase trafficking and processing by presenilins: Partial loss of function by familial Alzheimer's disease mutation. Proc. Natl. Acad. Sci. USA 2006, 103, 353-358.

21. Guibert, S.; Girardot, M.; Leveziel, H.; Julien, R.; Oulmouden, A. Pheomelanin coat colour dilution in French cattle breeds is not correlated with the TYR, TYRP1 and DCT transcription levels. Pigment. Cell Res. 2004, 17, 337-345.

22. Kushimoto, T.; Basrur, V.; Valencia, J.; Matsunaga, J.; Vieira, W.D.; Ferrans, V.J.; Muller, J.; Appella, E.; Hearing, V.J. A model for melanosome biogenesis based on the purification and analysis of early melanosomes. Proc. Natl. Acad. Sci. USA 2001, 98, 10698-10703.

23. Yi, R.; Fuchs, E., MicroRNA-mediated control in the skin. Cell Death Differ. 2010, 17, 229-235. 
24. Kim, A.; Yim, N.H.; Im, M.; Jung, Y.P.; Liang, C.; Cho, W.K.; Ma, J.Y. Ssanghwa-tang, an oriental herbal cocktail, exerts anti-melanogenic activity by suppression of the p38 MAPK and PKA signaling pathways in B16F10 cells. BMC Complement. Altern. Med. 2013, 13, 214-224.

25. Bellei, B.; Maresca, V.; Flori, E.; Pitisci, A.; Larue, L.; Picardo, M. P38 regulates pigmentation via proteasomal degradation of tyrosinase. J. Biol. Chem. 2010, 285, 7288-7299.

26. Kim, D.S.; Jeong, Y.M.; Park, I.K.; Hahn, H.G.; Lee, H.K.; Kwon, S.B.; Jeong, J.H.; Yang, S.J.; Sohn, U.D.; Park, K.C. A new 2-imino-1,3-thiazoline derivative, KHG22394, inhibits melanin synthesis in mouse B16 melanoma cells. Biol. Pharm. Bull. 2007, 30, 180-183.

27. Liu, Z.; Zhu, J.; Cao, H.; Ren, H.; Fang, X. MiR-10b promotes cell invasion through RhoC-AKT signaling pathway by targeting HOXD10 in gastric cancer. Int. J. Oncol. 2012, 40, 1553-1560.

28. Yu, X.; Li, Z.; Shen, J.; Wu, W.K.; Liang, J.; Weng, X.; Qiu, G. MicroRNA-10b promotes nucleus pulposus cell proliferation through RhoC-Akt Pathway by targeting HOXD10 in intervetebral disc degeneration. PLoS One 2013, 8, e83080.

29. Awgulewitsch, A. Hox in hair growth and development. Naturwissenschaften 2003, 90, 193-211.

30. Wu, J.H.; Yan, Z.W.; Husile; Zhang, W.G.; Li, J.Q. Hoxc13 and the development of hair follicle. Hereditas (Beijing) 2010, 32, 656-662.

31. Magnusson, M.; Brun, A.C.; Miyake, N.; Larsson, J.; Ehinger, M.; Bjornsson, J.M.; Wutz, A.; Sigvardsson, M.; Karlsson, S. HOXA10 is a critical regulator for hematopoietic stem cells and erythroid/megakaryocyte development. Blood 2007, 109, 3687-3696.

32. Yamaguchi, Y.; Passeron, T.; Hoashi, T.; Watabe, H.; Rouzaud, F.; Yasumoto, K.; Hara, T.; Tohyama, C.; Katayama, I.; Miki, T.; et al. Dickkopf 1 (DKK1) regulates skin pigmentation and thickness by affecting Wnt/beta-catenin signaling in keratinocytes. Fed. Am. Soc. Exp. Biol. 2008, 22, 1009-1020.

33. Yamaguchi, Y.; Passeron, T.; Watabe, H.; Yasumoto, K.; Rouzaud, F.; Hoashi, T.; Hearing, V.J. The effects of dickkopf 1 on gene expression and Wnt signaling by melanocytes: Mechanisms underlying its suppression of melanocyte function and proliferation. J. Investig. Dermatol. 2007, 127, 1217-1225.

34. Bolós, V.; Grego-Bessa, J.; de la Pompa, J.L. Notch signaling in development and cancer. Endocr. Rev. 2007, 28, 339-363.

35. Aubin-Houzelstein, G. Notch signaling and the developing hair follicle. Notch Signal. Embryol. Cancer 2012, 727, 142-160.

36. Osawa, M. Melanocyte stem cells. StemBook 2009, doi:10.3824/stembook.1.46.1.

37. Goding, C.R. Melanocytes: The new Black. Int. J. Biochem. Cell Biol. 2007, 39, 275-279.

38. Lang, D.; Mascarenhas, J.B.; Shea, C.R. Melanocytes, melanocyte stem cells, and melanoma stem cells. Clin. Dermatol. 2013, 31, 166-178.

39. Li, A. The biology of melanocyte and melanocyte stem cell. Acta Biochim. Biophys. Sin. 2014, 46, 255-260.

40. Osawa, M.; Fisher, D.E. Notch and melanocytes: Diverse outcomes from a single signal. J. Investig. Dermatol. 2008, 128, 2571-2574.

41. Kumano, K.; Masuda, S.; Sata, M.; Saito, T.; Lee, S.Y.; Sakata-Yanagimoto, M.; Tomita, T.; Iwatsubo, T.; Natsugari, H.; Kurokawa, M. Both Notch1 and Notch2 contribute to the regulation of melanocyte homeostasis. Pigment. Cell Melanoma Res. 2008, 21, 70-78. 
42. Schouwey, K.; Beermann, F. The Notch pathway, hair graying and pigment cell homeostasis. Histol. Histopathol. 2008, 23, 609-619.

43. Schouwey, K.; Larue, L.; Radtke, F.; Delmas, V.; Beermann, F. Transgenic expression of Notch in melanocytes demonstrates RBP-Jא-dependent signaling. Pigment. Cell Melanoma Res. 2010, 23, 134-136.

44. Sengupta, A.; Banerjee, D.; Chandra, S.; Banerji, S.K.; Ghosh, R.; Roy, R.; Banerjee, S. Deregulation and cross talk among Sonic hedgehog, Wnt, Hox and Notch signaling in chronic myeloid leukemia progression. Leukemia 2007, 21, 949-955.

45. Takacs-Vellai, K.; Vellai, T.; Chen, E.B.; Zhang, Y.; Guerry, F.; Stern, M.J.; Muller, F. Transcriptional control of Notch signaling by a HOX and a PBX/EXD protein during vulval development in C. elegans. Dev. Biol. 2007, 302, 661-669.

46. Vachtenheim, J.; Borovansky, J. "Transcription physiology" of pigment formation in melanocytes: Central role of MITF. Exp. Dermatol. 2010, 19, 617-627.

47. Margue, C.; Philippidou, D.; Reinsbach, S.E.; Schmitt, M.; Behrmann, I.; Kreis, S. New target genes of MITF-induced microRNA-211 contribute to melanoma cell invasion. PLoS One 2013, 8, e73473.

48. Mazar, J.; DeYoung, K.; Khaitan, D.; Meister, E.; Almodovar, A.; Goydos, J.; Ray, A.; Perera, R.J. The regulation of miRNA-211 expression and its role in melanoma cell invasiveness. PLoS One 2010, 5, e13779.

49. Friedländer, M.R.; Chen, W.; Adamidi, C.; Maaskola, J.; Einspanier, R.; Knespel, S.; Rajewsky, N. Discovering microRNAs from deep sequencing data using miRDeep. Nat. Biotechnol. 2008, 26, 407-415.

50. Friedländer, M.R.; Mackowiak, S.D.; Li, N.; Chen, W.; Rajewsky, N. MiRDeep2 accurately identifies known and hundreds of novel microRNA genes in seven animal clades. Nucleic Acids Res. 2012, 40, 37-52.

51. Anders, S.; Huber, W. Differential expression analysis for sequence count data. Genome Biol. 2010, 11, R106-R118.

52. Chen, C.; Ridzon, D.A.; Broomer, A.J.; Zhou, Z.; Lee, D.H.; Nguyen, J.T.; Barbisin, M.; Xu, N.L.; Mahuvakar, V.R.; Andersen, M.R. Real-time quantification of microRNAs by stem-loop RT-PCR. Nucleic Acids Res. 2005, 33, e179.

53. Livak, K.J.; Schmittgen, T.D. Analysis of relative gene expression data using real-time quantitative PCR and the $2^{-\Delta \Delta C t}$ method. Methods 2001, 25, 402-408.

54. Da Wei Huang, B.T.S.; Lempicki, R.A. Systematic and integrative analysis of large gene lists using DAVID bioinformatics resources. Nat. Protocol. 2008, 4, 44-57.

55. Sherman, B.T.; Lempicki, R.A., Bioinformatics enrichment tools: paths toward the comprehensive functional analysis of large gene lists. Nucleic Acids Res. 2009, 37, 1-13.

(C) 2014 by the authors; licensee MDPI, Basel, Switzerland. This article is an open access article distributed under the terms and conditions of the Creative Commons Attribution license (http://creativecommons.org/licenses/by/3.0/). 